\title{
Psychological and Physiological Predictors of Lipids in Black Males
}

\author{
Ernest H. Johnson, ${ }^{1,5}$ Phillip Collier, ${ }^{2}$ Pietro Nazzaro, ${ }^{3}$ \\ and Douglas C. Gilbert ${ }^{4}$
}

Accepted for publication: June 26, 1991

The association between lipids and both psychological and physiological
measures were examined in this study of healthy black males. The results
revealed that certain psychological measures, namely, State and Trait Curiosity
and Trait Anger, explained a significant proportion of the variance in high-
density lipoproteins (HDL), low-density lipoproteins (LDL), and triglycerides.
Although psychological factors accounted for a significant proportion of the
variance in lipids (29\% for HDL, $25 \%$ for LDL, $64 \%$ for LDL/HDL, $29 \%$
for triglyceride), the amount of explained variance was significantly increased
by the inclusion of both psychological and physiological variables in the regres-
sion equation. However, neither of the psychological variables explained any
of the variance for total cholesterol when physiological variables were included
in the regression analysis. The overall pattern of the findings suggests that black
males who are at increased risk for elevated lipid levels may be identified by
their level of mental vigilance, the frequency at which their anger is experienced,
and the presence of other traditional risk factors.

KEY WORDS: lipids; predictors, psychological, physiological; black males.

This work was supported by NHLBI Grant RO1-HL38424 to Dr. Johnson, who completed this study at the Division of Hypertension at the University of Michigan Medical Center.

${ }^{1}$ Department of Family Medicine, Morehouse School of Medicine, 505 Fairburn Road, S. W., Southwest Professional Medical Building, Atlanta, Georgia 30331-2099.

${ }^{2}$ University of Michigan Medical School, Ann Arbor, Michigan.

${ }^{3}$ Bari University, Bari, Italy.

${ }^{4}$ Department of Psychology, University of Houston, Houston, Texas 77204.

${ }^{5}$ To whom correspondence should be addressed. 


\section{INTRODUCTION}

Elevated levels of total and low levels of high-density lipoprotein (HDL) cholesterol have been clearly recognized as one of the important risk factors for coronary heart disease (CHD) in black and white Americans (Gillum, 1984; Gillum and Grant, 1984; Grundy and Winston, 1989; Becker, 1989; Kowalski, 1989; King, 1989). The extent of coronary atherosclerosis and CHD has been found to be positively related to total cholesterol (Kannel, Castelli and Gordon, 1979), low-density lipoprotein cholesterol (LDL; Kannel et al., 1979), triglycerides (Lippel et al., 1981), and the ratio of LDL to HDL (Kannel et al., 1979) in the United States and other countries (Keys, 1970; Wilson, 1989). Recently, the search for factors that are related to elevated lipid levels has been broadened to include stress and emotional factors, primarily because known risk factors such as age, weight, blood pressure, diet, exercise, smoking, and alcohol intake do not account for all of the variance in elevated lipid levels (Dimsdale and Herd, 1982; Van Doornen and Orlebeke, 1982; Kannel and Eaker, 1986). Although variations in lipid levels are due partly to dietary factors, psychological factors such as stress, mood, and personality also appear to be involved (Dimsdale and Herd, 1982; Van Doornen and Oelebeke, 1982). An early study by Jenkins et al. (1969) showed that Type A behavior and its hostility dimension was significantly related to serum cholesterol.

The relationship between emotions and cholesterol is beginning to receive added attention from researchers and the general public. It appears that most people in the United States are aware of the link between heart disease and high cholesterol, but only recently has research shown strong indications that emotional and personality factors contribute to the rise in cholesterol levels (Weidner et al., 1989; Lundberg et al., 1989; Waldstein et al., 1990). For example, a study reported in 1987 by Weidner and associates examined the relationship of hostility and Type A behavior to plasma lipids (specifically, cholesterol) and lipoproteins in a community sample of women and men from Portland, Oregon. Overall, the pattern of results suggests that high levels of hostility among persons who possess strong type A characteristics is related to elevated levels of plasma total and LDL cholesterol in both men and women.

In another recent study, Waldstein and associates (1990), at the University of Pittsburgh, examined the relationship of several dimensions of anger and its expression to fasting total cholesterol and lipoprotein concentrations. The subjects for this study were 29 healthy white males (average age, 24) who completed the Spielberger Trait Anger and Anger Expression Scales (Spielberger et al., 1985; Spielberger, 1988); the latter instrument subsumes three subscales termed 
Anger-Out, Anger-In, and Anger-Control/Reflection. The results of this study revealed a significant positive correlation between Anger-Out and HDL $(r=.46, \mathrm{p}<.01)$. Correlations between Anger-Out and both HDL2 $(r=.34, p<.07)$ and HDL3 $(r=.34, p<.07)$ were marginally significant. In addition, Trait Anger (i.e., the frequent experience of anger) correlated significantly with HDL3 $(r=.44, p<.02)$ and marginally with total HDL $(r=.34, p<.07)$. Finally, Anger-Control/Reflection was inversely related to total HDL $(r=-.37, p<.05)$ and HDL3 $(r=-.34, p<.07)$. These results demonstrate a moderate relationship between fasting HDL cholesterol concentrations and both the experience (Trait Anger) and the outward (Anger-Out) expression of anger. On the other hand, a reluctance to acknowledge or express anger (Anger-Control/Reflection) is associated with a relatively lower (i.e., potentially deleterious) HDL concentration.

Although evidence of a moderate association between anger and cholesterol concentrations (Weidner et al., 1989; Lundberg et al., 1989; Waldstein et al., 1990) as well as a strong association between anger and hypertension (Diamond, 1982; Chesney and Rosenman, 1985) is mounting, there has been no investigation of the relationships between emotional factors and cholesterol among blacks. The purpose of the present inquiry is to describe the relationship between emotional factors and plasma total cholesterol, HDL, LDL, and triglycerides among a sample of black males. This study also examines the extent to which cholesterol and lipid levels are related to other risk factors for CHD. Finally, the present inquiry will determine the relative independent contribution of both emotional and physiological factors to the prediction of plasma lipids.

\section{METHODS}

\section{Subjects and Measures}

The data for this report are based on information obtained from 38 healthy black men who were recruited as part of a larger study of the "Behavioral Factors in the Etiology of Hypertensive Blacks" that was being conducted at the division of Hypertension at the University of Michigan Medical Center. To be considered a subject for this study, all individuals had to meet the following criteria: (1) not having heart disease and/or diabetes, (2) not having hypertension or kidney disease, (3) not taking prescription medication, and (4) not frequently using a relaxation technique. A brief physical examination of the subjects was 
performed by a physician at the Hypertension Clinic a few days before the physiological measures were obtained. All subjects fasted for $12 \mathrm{hr}$ and abstained from smoking cigarettes, consuming alcohol, or ingesting caffeinated beverages. Following the physical examination, blood was drawn for the lipid determinations without stasis into tubes containing dry ethylenediaminetetraacetic acid (EDTA) by a clinic nurse. The samples were transported to the pathology laboratory, where the plasma was separated by centrifugation and then analyzed. All subjects were compensated for participating in the investigation.

\section{Psychologic Measures}

Anger Expression Scale $(A X)$. The AX was developed by Spielberger et al. (1985) and Johnson et al. (1987a, b) to measure the frequency with which anger is expressed. The AX yields a total scale score and AX/Anger-In (suppressed anger), AX/Anger-Out, and AX/Anger-Control/Reflection subscale scores. A new subscale that measures the degree of fear and anxiety (Anger-Fear) associated with the expression of anger has been added to the AX. The internal consistency of the AX total scale and its subscales, as measured by Cronbach's alpha coefficients, ranged from .70 to .84 for the subjects in previous investigations (Spielberger et al., 1985; Johnson et al., 1987a, b; Spielberger, 1988).

State-Trait Personality Inventory (STPI). The STPI was developed by Spielberger et al. (1979) to measure anger, anxiety, and curiosity as emotional states and personality traits. The STPI State Scale consists of three 10 -item subscales for measuring the intensity of anger (State Anger), anxiety (State Anxiety), and curiosity (State Curiosity), while the Trait Scale requires the respondents to report the frequency that they have experienced feelings of anger (Trait Anger), anxiety (Trait Anxiety), and curiosity (Trait Curiosity).

State Anger Reaction Scale (S-Anger/RX). The S-Anger/RX was developed to measure the intensity of anger experienced in stressful and frustrating social situations (Johnson et al., 1987a, b). The S-Anger/RX yields a total scale score and two subscales for measuring angry responses to time pressure (TP) and evaluative-threatening (ET) situations. The internal consistency of the S-Anger/RX total scale and its TP and ET subscales as measured by alpha coefficients ranged from .81 to .87 for the subjects in the previous studies (Johnson et al., 1987a, b). Item remainder correlations for the TP and ET subscales ranged from .42 to .67 . 


\section{Physiological Measures}

Measurements of heart rate (HR) and systolic and diastolic blood pressure (BP) were obtained during a brief physical examination of the subject. Forearm blood flow (FABF; $\mathrm{ml} / 100 \mathrm{ml}$ forearm volume/min) was measured using mercury-in-Silastic strain gauge plethysmography with intermittent venous occlusion in the left arm by a blood pressure cuff concurrent with isolation of the wrist and hand circulation by a second cuff (Egan and Julius, 1985; Egan et al., 1988).

Forearm vascular resistance was calculated by dividing mean arterial blood pressure (MAP) by forearm blood flow. The left forearm was supported above heart level. The strain gauge was placed around the forearm, approximately $7 \mathrm{~cm}$ below the olecranon. Sixty seconds before FABF determination, hand blood flow was arrested by a pediatric-sized cuff at the wrist, inflated to suprasystolic pressure. A second cuff on the arm was subsequently inflated to $40-50 \mathrm{mmHg}$ for $10-15 \mathrm{sec}$ and deflated for $5 \mathrm{sec}$ for each of four cycles. Forearm blood flow was calculated from the mean vertical deflection per minute on the four tracings divided by the $1 \%$ electrical calibration signal.

Minimum FAVR was calculated as MAP/maximum FABF. Maximum FABF was measured after $10 \mathrm{~min}$ of ischemic forearm exercise. Maximum flow was determined from the mean of the six highest flow curves obtained in the $60-90 \mathrm{sec}$ immediately following the ischemic period.

The plasma lipid assessment consisted of determination of plasma total cholesterol, HDL, LDL, and plasma triglycerides. Total serum cholesterol and triglycerides were determined enzymatically, while HDL was assayed by a precipitation procedure (Warnick and Ables, 1978). LDL was arithmetically calculated from HDL, total cholesterol, and triglycerides using the Friedewald equation (Friedwald et al., 1972). The ratio of LDL to HDL was also derived because it provides a good estimate of the joint effect of these opposing cholesterol-lipoprotein fractions (Kannel et al., 1979). The assays were conducted in a laboratory at the University of Michigan Medical Center that follows the guidelines of the Lipid Research Clinic Programs (1975).

\section{Protocol}

Each subject was tested individually in a small, quiet laboratory by the same research assistants. All subjects were tested between $8 \mathrm{AM}$ and 12 noon after having fasted overnight, and abstained from smoking cigarettes, consuming alcohol, or ingesting caffeinated or decaffeinated coffee or 
beverages since dinner the previous night. All studies were completed with the subjects in a recumbent position with their heads on pillows. Upon arrival at the Hypertension Research Laboratory, each subject was greeted by the assistant and written informed consent was obtained. After written consent was obtained, the subject completed the psychological questionnaires including the state scales of the STPI to determine their level of State-Anxiety, State-Anger, and State-Curiosity prior to the measurement of physiological factors. After the questionnaires were completed, the laboratory assistant attached the BP and plethysmographic apparatus and remained in the room with the subject to measure $B P, H R$, and FABF during the resting (baseline) period. After the BP and plethysmographic apparatus was connected, a needle was placed in a vein on the right forearm for the purpose of drawing blood samples for measuring plasma catecholamines. Once all the equipment was adjusted, the subject lay supine for $30 \mathrm{~min}$ listening to quiet music while BP and HR were obtained every 2 min. FABF was measured after 10 min of rest and again at the 30-min mark. Blood for plasma catecholamine determination was drawn $20 \mathrm{~min}$ into the 30 -min rest period and the sample was assayed by a single isotope radioenzymatic method (Peuler and Johnson, 1977). Other cardiovascular data (not reported in this paper) was collected in response to a series of stressful laboratory tasks that lasted for approximately $60 \mathrm{~min}$. At the end of this period the subjects engaged in $10 \mathrm{~min}$ of ischemic forearm exercise so that maximum FABF could be measured.

\section{RESULTS}

The demographic, physiological, and average psychological scales scores for the black males in this study are presented in Table I. In general, the black males in this sample had average blood pressure and weight for their age and height. A large percentage of the men smoked cigarettes (21\% were current smokers) and drank alcohol (62\% were current drinkers). Of the males who are current smokers, $9 \%$ smoked daily - at least 3 cigarettes. Of the current drinkers, $3 \%$ drank on a daily basis - at least 3 drinks. Plasma catecholamines, lipids, and psychological measurements were within normal limits.

The data were analyzed in the following manner. For each lipid measure separately, correlations were used to determine the linear associations between lipids and both the psychological and the physiological variables. Multiple regression assessed the strength of the associations and a forward stepwise regression approach was employed to determine which significant psychological and physiological variables explained unique proportions of 
Table I. Demographic, Physiological, and Average Psychological Scale Scores for Black Males

\begin{tabular}{|c|c|c|}
\hline Variable & Mean & STD \\
\hline Age (yr) & 29 & 8 \\
\hline Weight (lb) & 173 & 31 \\
\hline Height (in) & 70 & 2 \\
\hline SBP (mm Hg) & 125 & 14 \\
\hline $\mathrm{DBP}(\mathrm{mm} \mathrm{Hg})$ & 83 & 12 \\
\hline HR (bpm) & 75 & 12 \\
\hline $\mathrm{M}-\mathrm{FABF}(\mathrm{ml} / \mathrm{dl} / \mathrm{min})$ & 36 & 11 \\
\hline M-FAVR $[\mathrm{mm} \mathrm{Hg} /(\mathrm{ml} / \mathrm{dl} / \mathrm{min})]$ & 3 & 1 \\
\hline Plasma NE (pg/ml) & 265 & 138 \\
\hline Plasma EPI $(\mathrm{pg} / \mathrm{ml})$ & 62 & 54 \\
\hline Smokers (\%) & $56^{a}$ & - \\
\hline Drinkers (\%) & $86^{b}$ & - \\
\hline Anger-Control/Refl. & 41 & 7 \\
\hline Anger-Out & 16 & 3 \\
\hline Anger-In & 17 & 4 \\
\hline Anger-Fear & 7 & 2 \\
\hline Evaluation Threat & 24 & 5 \\
\hline Time Pressure & 18 & 4 \\
\hline T-Anxiety & 23 & 4 \\
\hline T-Curiosity & 27 & 5 \\
\hline T-Anger & 21 & 5 \\
\hline S-Anxiety & 18 & 6 \\
\hline S-Curiosity & 23 & 5 \\
\hline S-Anger & 12 & 4 \\
\hline Cholesterol (mg/dl) & 177 & 29 \\
\hline HDL (mg/dl) & 58 & 18 \\
\hline $\mathrm{LDL}$ (mg/dl) & 106 & 27 \\
\hline Triglycerides (mg/dl) & 99 & 50 \\
\hline $\mathrm{LDL} / \mathrm{HDL}(\mathrm{mg} / \mathrm{dl})$ & 2.10 & 0.87 \\
\hline
\end{tabular}

${ }_{35 \%}$ ex-smokers $+12 \%$ sometimes/not daily $+6 \%$ daily $/ 3-4$ cigarettes $+3 \%$ daily/half-pack. ${ }^{b} 24 \%$ ex-drinkers $+38 \%$ very light/infrequent $+21 \%$ moderate/not daily $+3 \%$ heavy $/ 3-4$ drinks per day.

the variance in lipids. The first set of regression analyses determined the extent to which the psychological factors alone explained the variance in lipids. A similar set of analyses was conducted to determine the extent that the physiological factors explained the variance in lipids, while the final set of analyses determined the extent to which psychological and physiological factors jointly explained the variance in lipids.

Table II presents the correlations between the lipid measures and both the psychological and the physiological measures. For cholesterol, State-Curiosity was the only variable that showed a significant positive cor- 
Table II. Correlations Between Psychological Factors and Lipids in Black Males

\begin{tabular}{lccccc}
\hline \multicolumn{1}{c}{ Variables } & Cholesterol & HDL & LDL & LDL/HDL & Triglycerides \\
\hline Anger-Control/Refl. & .02 & -.12 & .15 & -.24 & .08 \\
Anger-Out & .01 & -.16 & -.06 & .20 & .19 \\
Anger-In & -.14 & .14 & -.32 & .01 & -.26 \\
Anger-Fear & -.24 & .09 & -.29 & -.05 & -.27 \\
Evaluation Threat & -.07 & .23 & -.13 & -.04 & -.04 \\
Time Press & .13 & .21 & -.08 & .27 & -.01 \\
T-Anxiety & -.04 & .20 & -.31 & .19 & -.26 \\
T-Curiosity & .22 & -.29 & $.58^{* * *}$ & -.05 & $.64^{* * *}$ \\
T-Anger & .01 & -.02 & -.11 & $.41^{* *}$ & $.21^{* *}$ \\
S-Anxiety & -.07 & -.05 & -.08 & .21 & -.01 \\
S-Curiosity & $.33^{*}$ & -.31 & $.45^{* *}$ & $.27^{* *}$ & $.62^{* * *}$ \\
S-Anger & -.04 & -.09 & -.07 & .25 & .01 \\
& & & & & \\
Age & $.49^{* * *}$ & -.03 & $.52^{* * * *}$ & .29 & .28 \\
Weight & -.07 & -.23 & .02 & -.16 & -.03 \\
Height & $-.35^{*}$ & -.22 & -.17 & -.17 & -.03 \\
SBP & .19 & -.17 & .02 & .12 & .14 \\
DBP & .14 & $-.31^{*}$ & .19 & .07 & .18 \\
HR & .11 & -.22 & .29 & -.13 & .19 \\
M-FABF & -.29 & $-.33^{*}$ & -.31 & .17 & .09 \\
M-FAVR & $.46^{* * *}$ & .17 & $.53^{* * *}$ & -.09 & .11 \\
Plasma NE & .15 & .23 & .05 & .23 & .02 \\
Plasma EPI & $.43^{* *}$ & $.38^{* *}$ & .15 & .07 & -.17 \\
Smoking & .19 & .01 & .17 & .20 & .10 \\
Drink & .14 & .19 & -.25 & .24 & -.24 \\
\hline
\end{tabular}

${ }^{*} p<.10$.

${ }^{* *} p<.05$.

relation, and none of the variance could be explained by the regression model. For HDL, neither of the psychological variables showed significant correlations, but the results of the regression analysis indicated that two variables (Time Pressure, State Curiosity) accounted for $29 \%$ of the variance. In contrast, curiosity (State and Trait) showed a significant positive correlation with LDL cholesterol, and State Curiosity alone accounted for $25 \%$ of the LDL variance. For the LDL/HDL ratio, two variables (Trait Anger, State Curiosity) showed significant positive correlations; together they accounted for $64 \%$ of the variance. Finally, three variables (Trait Curiosity, Trait Anger, State Curiosity) showed significant positive correlations with triglycerides, but Trait Anger was the only variable to explain a significant proportion (29\%) of the variance.

The bottom portion of Table II presents the correlations between the physiological and the lipid measures. For total cholesterol, four variables [age, height, M-FAVR, and plasma epinephrine (EPI)] showed significant correlations. The results of the stepwise regression analysis for 
Table III. Stepwise Regression Using Physiological and Psychological Variables as Predictors of Lipids for Black Males

\begin{tabular}{|c|c|c|c|c|c|c|c|c|c|}
\hline \multicolumn{2}{|c|}{ Cholesterol } & \multicolumn{2}{|c|}{ HDL } & \multicolumn{2}{|c|}{ LDL } & \multicolumn{2}{|c|}{ LDL/HDL } & \multicolumn{2}{|c|}{ Triglycerides } \\
\hline $\begin{array}{l}\text { EPI } \\
\text { Height } \\
\text { Weight } \\
\text { SBP }\end{array}$ & $\begin{array}{l}(.37)^{* *} \\
(.18)^{* *} \\
(.19)^{* *} \\
(.06)^{*}\end{array}$ & $\begin{array}{l}\text { SCUR } \\
\text { Height } \\
\text { EPI } \\
\text { SBP }\end{array}$ & $\begin{array}{l}(.17)^{*} \\
(.16)^{*} \\
(.16)^{*} \\
(.16)^{*}\end{array}$ & $\begin{array}{l}\text { TCUR } \\
\text { Drink } \\
\text { Age } \\
\text { Cigarettes } \\
\text { SCUR }\end{array}$ & $\begin{array}{l}(.29)^{* *} \\
(.20)^{* *} \\
(.23)^{* *} \\
(.09)^{* *} \\
(.05)^{* *}\end{array}$ & $\begin{array}{l}\text { SCUR } \\
\text { TANG } \\
\text { EPI } \\
\text { Cigarettes } \\
\text { NE } \\
\text { Weight }\end{array}$ & $\begin{array}{l}(.51)^{* *} \\
(.14)^{*} \\
(.13)^{*} \\
(.09)^{*} \\
(.06)^{*} \\
(.03)^{*}\end{array}$ & TANG & $(.29)^{* *}$ \\
\hline Total $R^{2}$ & .80 & & .65 & & .97 & & .96 & & .29 \\
\hline
\end{tabular}

total cholesterol indicated that four variables (weight, height, systolic blood pressure, and plasma epinephrine) accounted for $82 \%$ of the total variance. The first to enter the equation was plasma epinephrine, which explained $29 \%$ of the variance. For HDL, two variables (plasma epinephrine, systolic blood pressure) helped to explain $27 \%$ of the HDL variance. For LDL, age and M-FAVR showed the most significant positive correlations, but neither of these variables accounted for the 74\% of the LDL variance. In contrast, four variables [heart rate, M-FABF, plasma norepinephrine (NE), plasma epinephrine] that were not linearly associated with LDL accounted for the variance. Finally, neither of the physiological variables was significantly correlated with the LDL/HDL ratio or triglycerides.

Table III presents the results of the stepwise multiple regression analyses which determined the extent to which psychological and physiological measures contributed to the prediction of lipids. The results for cholesterol showed that neither of the psychological variables accounted for the total cholesterol variance. However, four physiological variables accounted for $80 \%$ of the variance. The overall pattern of the findings indicates that higher cholesterol levels among black males are characterized by higher epinephrine and systolic blood pressure levels and lower weight. For each of the other lipid measures psychological factors played a prominent role in accounting for the variance in lipids. The results for HDL indicated that State Curiosity was the first variable to enter the regression equation. State Curiosity together with three physiological variables (height, epinephrine, systolic blood pressure) explained $65 \%$ of the HDL variance. For LDL, Trait Curiosity was the first variable to enter the regression equation and, together with three traditional risk factors (alcohol intake, age, smoking) and State Curiosity, explained $97 \%$ of the variance in LDL. The ratio of LDL/HDL was 
explained primarily by two psychological variables (State Curiosity, Trait Anger) that accounted for approximately $65 \%$ of the variance. Even though four physiological variables (plasma epinephrine, smoking, plasma norepinephrine, weight) entered the regression equation, the variance explained $(31 \%)$ was not as great as that for the psychological variables. For triglycerides, Trait Anger was the sole predictor and accounted for $29 \%$ of the variance.

\section{DISCUSSION}

It has been demonstrated here that certain psychological and physiological factors are associated with lipids among black males. Although there is a growing body of literature indicating that psychological and stressrelated factors (Rosenman et al., 1976; Mathews et al., 1977; Barefoot et al., 1983; Dembroski et al., 1985; Schekelle et al., 1983) are related to CHD, there appears to be no pathway upon which all researchers agree. Perhaps the associations among lipids, stress, and CHD among blacks would be understood better if research focused on determining the joint contribution of psychological stress and traditional physiological factors to the prediction of elevated lipid levels. In this regard, the present investigation revealed that certain psychological measures, namely, State and Trait Curiosity and Trait Anger, were significantly associated with HDL, LDL, and triglycerides. However, the amount of explained variance was significantly increased by the inclusion of physiological variables in the regression equation. The exception to this was triglycerides; neither of the physiological variables explained any of the variance in triglycerides.

The reasons for the strong association between State and Trait Curiosity and lipids are unclear and there are no published findings with which to compare the results. However, the results of the present inquiry are inconsistent with those of Waldstein et al. (1990), who observed significant associations between HDL cholesterol and measures of the experience (Trait Anger) and expression (Anger-Out) of anger for their sample of white males. A plausible explanation for the inconsistency of results is that subjects in the present inquiry were black, while those in the Waldstein study were white. On the other hand, the failure to find significant associations between HDL and anger might truly mean that anger-related variables are not important predictors of lipid levels among blacks. On the other hand, a measure of the disposition to experience intense anger when provoked (Trait Anger) was a significant predictor of triglycerides and the ratio of LDL to HDL for black males in the present inquiry. Anger might contribute to the atherosclerotic process by keeping triglycerides and LDL/HDL ratio elevated. It is conceivable, for ex- 
ample, that individuals who frequently experience intense anger also experience high arousal situations which increase the release of catecholamines as a result of sympathetic nervous system activation. In the long run, the excessive release of catecholamines might contribute to lipid mobilization and atherosclerosis. However, confirmation of this scenario must come from future studies.

As indicated above, there are no published studies with which to compare the results for State and Trait Curiosity; however, the relations found in the present inquiry are quite reasonable. For example, HDL cholesterol was associated with a low level of State Curiosity, while LDL cholesterol was associated with a high level of both State and Trait Curiosity. The overall pattern of these findings suggests that low levels of mental vigilance and concerns about what is going on within one's environment are associated with HDL cholesterol levels. In contrast, high levels of mental vigilance and suspiciousness about one's environment are associated with LDL cholesterol. Such findings are particularly interesting in view of the fact that black American males are known to suffer from greater exposure to life stress and encounter more problem situations and difficulties that require continuous mental and psychological adaptation (most often with inadequate resources) than any other racial groups (Health Resources Administration, 1980; National Center for Health Statistics, 1979; Broman and Johnson, 1988). It may also be the case that the measures of State and Trait Curiosity are markers and predictors of other personality factors such as suspiciousness, cynicism, and paranoia. Because black men have been historically undervalued and viewed with great suspiciousness and mistrust, it would only seem reasonable to assume that some of the emotional and mental sequelae associated with their everyday functioning would include heightened mental vigilance and suspiciousness. In fact, it has been argued that to be suspicious of one's environment is a black norm that is necessary for the survival of black people (Grier and Cobb, 1969).

Although it is not understood how emotional factors influence lipid levels, enhanced mental vigilance and suspiciousness of one's environment may play an important role in triggering the transport of fat out of storage and into the bloodstream while people are under stress. Perhaps black men who cope with environmental demands with increased mental and emotional vigilance mobilize more fat and have a more exaggerated physiological and neuroendocrine response to stressful situations than do others. Eventually, this could drive up lipid levels and create more opportunities over time for the buildup of plaque in the arteries. It should be noted that these notions are in line with the body of research (Engel, 1977; Brod, 1963; Zanchetti et al., 1972; Obrist, 1981) indicating that when cognitive appraisal of situations results in a sense of threat and danger or the need for continuous mental 
efforts to cope with the situations, a characteristic defense reaction or "fight/flight" pattern is observed.

There are a number of methodological problems inherent in the present investigation, which makes it a bit difficult to draw firm conclusions. First, the data are based on a small, nonrandom sample of only 38 black males and the findings might not generalize to large black populations. We have no empirical way to reconcile this problem. However, it should be noted that the lipid and blood pressure levels for black males in the present study are remarkably similar to those of a subset of blacks who participated in the Lipid Research Clinics Program Prevalence Study (Ekelund et al., 1990). For example, HDL cholesterol was $55.2 \mathrm{mg} / \mathrm{dl}$ for subjects in the Lipid Clinics Study and $58 \mathrm{mg} / \mathrm{dl}$ for subjects in the present study. Similarly, triglycerides were 102.8 for blacks in the Lipid Clinics Study and $99 \mathrm{mg} / \mathrm{dl}$ for subjects in the present study. Finally, blood pressure was $126.9 / 85.2 \mathrm{~mm} \mathrm{Hg}$ for subjects in the Lipids Clinics Study and $125 / 83 \mathrm{~mm} \mathrm{Hg}$ for subjects in the present study. While it may be true that the sample for the present study is small, we await future research to determine whether the pattern of findings is not generalizable to other black populations. We also recognize that the lipid values were relatively within the normal range and that it would be preferable to conduct a similar set of analyses in groups that differed by high vs. low lipid levels. While there is good evidence that genetic factors contribute to lipid levels (Sing et al., 1980), we did not measure genetic factors in this study and it is possible that the findings of the present inquiry may, to some extent, be attributable to or mediated by genetic differences among individuals.

In conclusion, the data are supportive of an association between psychological factors and lipids for black males, although it should be noted that a larger proportion of the variance in lipids could be explained by the inclusion of both physiological and psychological variables in the regression equation. Because of the cross-sectional nature of the data, definitive statements cannot be made as to whether psychological factors precede or are a consequence of elevated lipid levels. However, it is our belief that the pendulum swings in the other direction, with frequent experience of anger and mental vigilance about problems in one's environmental milieu being the initial event and elevated lipid levels following. To understand better the etiologic significance of the pattern of findings in this inquiry, it would appear necessary that future research efforts be directed at identifying the physiologic abnormalities underlying the relationship among lipids, mental vigilance, and anger. It is conceivable that the physiological profiles may be different for black and white subjects as well as for males and females. Consequently, studies of this nature may lead to a greater understanding of the role of emotional and behavioral factors in the development of CHD and its sequelae in black and white Americans. 


\section{REFERENCES}

Barefoot, J. C., Dahlstrom, W. S., and Williams, R. B. (1983). Hostility, CHD incidence, and total mortality: A 25-year follow-up study of 255 physicians. Psychosom. Med. 1983; 45: 59-63.

Becker, G. L. (1987). Heart Smart, Fireside.

Brod, J. (1963). Hemodynamic basis of acute pressor reactions and hypertension. Br. Heart J. 25: $227-245$.

Broman, C. L., and Johnson, E. H. (1988). Anger expression and life stress among blacks: Their role in physical health. JNMA 80: 1329-1334.

Dembroski, T. M., MacDougall, J. M., Williams, R. B., Haney, T. L., and Blumenthal, J. A. (1985). Components of Type A behavior, hostility, and anger-in: Relationship to angiographic findings. Psychosom. Med. 47: 219-233.

Dimsdale, J. E., and Herd, A. (1982). Variability of plasma lipids in response to emotional arousal. Psychosom. Med. 44: 413-430.

Egan, B., and Julius, S. (1985). Vascular hypertrophy in borderline hypertension: Relationship to blood pressure and sympathetic drive. Clin. Exp. Hypertens. A 7: 242-255.

Egan, B., Schork, N., Panis, R., and Hinderliter, A. (1988). Vascular structure enhances regional resistance responses in mild hypertension. J. Hypertens. 6: 41-48.

Ekelund, L., Suchindran, C. M., Karon, J. M., McMahon, R. P., and Tyroler, H. A. (1990). Black-white differences in exercise blood pressure - The Lipid Research Clinics Program Prevalence study. Circulation 31: 1568-1574.

Engel, G. L. (1971). Sudden and rapid death during psychological stress. Ann. Intern. Med. 74: $771-782$.

Friedwald, W. T., Levy, R. I., and Frederickson, D. S. (1972). Estimation of the concentration of LDL-C in plasma without the use of preparative ultracentrifuge. Clin. Chem. 18: 499-502.

Gillum, R. F. (1984). Coronary heart disease in black populations. I. Mortality and morbidity. Am. Heart J. 104: 839-851.

Gillum, R. F., and Gant, C. T. (1984). Coronary heart disease in black populations. II. Risk factors. Am. Heart J. 104: 852-860.

Grier, W. H., and Cobb, P. M. (1969). Black Rage, Batham Books, New York.

Grundy, S., and Winston, M. (1989). American Heart Association Low-Fat, Low-Cholesterol Cookbook, Times Books/Random House, New York.

Health Resources Administration (1979). Health of the Disadvantaged. Public Health Service, DHHS publication No. (HR) 80-633, Government Printing Office, Washington, DC.

Jenkins, C. D., Hames, C. G., and Zyzanski, S. J. (1969). Psychological traits and serum lipids. Psychosom. Med. 31: 115-128.

Johnson, E. H., and Broman, C. L. (1987). The relationship of anger expression to health problems among black Americans in a national survey. J. Behav. Med. 10: 103-116.

Johnson, E. H., Spielberger, C. D., Worden, T. J., and Jacobs, G. A. (1987a). Emotional and familial determinants of elevated blood pressure in black and white adolescent males. $J$. Psychosom. Res. 31: 287-300.

Johnson, E. H., Schork, N. J., and Spielberger, C. D. (1987b). Emotional and familial determinants of elevated blood pressure in black and white adolescent females. J. Psychosom. Res. 31: 731-741.

Kannel, W. B., and Eaker, E. D. (1986). Psychosocial and other features of coronary heart disease. Insights from the Framingham Study. Am. Heart J. 112: 1066-1073.

Kannel, W. B., Castelli, W. P., and Gordon, T. (1979). Cholesterol in the prediction of atherosclerotic disease: New perspectives based on the Framingham Study. Ann. Intern. Med. 90: 85-91.

Keys, A. (1970). Coronary heart disease in seven countries. Circulation 41 (Suppl. 1): 1-99.

King, P. (1989). Double jeopardy: Cholesterol and Type A. Minding your health - Mind over cholesterol. Psychol. Today Sept.: 26.

Kowalski, R. E. (1989). The 8-Week Cholesterol Cure, Harper and Row, New York. 
Lipid Research Clinic Programs (1975). Manual of Laboratory Operations. Vol. 1. Lipid and Lipoprotein Analysis, DHEW (Publ. No. NIH) 75-628.

Lippel, K., Tyroler, H., Eder, H., Gotto, A., and Vahouny, G. (1981). Meeting summary: relationship of hypertriglygeridemia to atherosclerosis. Atheriosclerosis 1: 406-417.

Lundberg, U., Hedman, M., Melin, B., and Frankenhaeuser, M. (1989). Type A behavior in healthy males and females as related to physiological reactivity and blood lipids. Psychosom. Med. 51: 113-22.

Mathews, K., Glass, D., Rosenman, R., and Bortner, R. (1977). Competitive drive, pattern $\mathrm{A}$, and coronary heart disease: A further analysis of some data from the Western Collaborative Group Study. J. Chron. Dis. 30: 489-498.

National Center for Health Statistics (1979). Health, United States, Public Health Service, DHHS No. (PHS) 80-1232, Government Printing Office, Washington, DC.

Obrist, P. A. (1981). Cardiovascular Psychophysiology: A Perspective, Plenum Press, New York.

Peuler, J. D., and Johnson, G. A. (1977). Simultaneous single isotope radioenzymatic assay of plasma norepinephrine, epinephrine, and dopamine. Life Sci. 21: 625-636.

Shekelle, R. B., Gayle, M., Ostfeld, M. A., and Paul, O. (1983). Hostility, risk of CHD, and mortality. Psychosom. Med. 45: 109-114.

Sing, C. F., Orr, J. D., and Moll, P. J. (1980). Review of factors that predict serum cholesterol in general population. In Lauer, R. M. and Shekelle, R. B. (eds.), Childhood Prevention of Atherosclerosis and Hypertension, Ravern Press, New York, pp. 87-97.

Spielberger, C. D. (1988). State-Trait Anger Expression Inventory (STAXI), Psychologic and Assessment Resources.

Spielberger, C. D., Jacobs, G. A., Barker, L., et al. (1979). Preliminary manual for the statetrait personality inventory (STPI). Center for Research in Behavioral Medicine and Health Psychology, University of South Florida, Tampa.

Spielberger, C. D., Jacobs, G. A., Russell, S., and Crane, R. (1983). Assessment of anger: The State-Trait Anger Scale. In Butcher, J. N. and Spielberger, C. D. (eds.), Advances in Personality Assessment, Vol. 2, LEA, Hillsdale, NJ.

Spielberger, C. D., Johnson, E. H., Russell, S. F., Crane, R., Jacobs, G. A., and Worden, T. J. (1985). The experience and expression of anger: Construction and validation of an anger expression scale. In Chesney, M. A., and Rosenman, R. H. (eds.), Anger and Hostility in Cardiovascular and Behavioral Disorders, McGraw-Hill, New York.

Van Doornen, L. J. P., and Orlebeke, J. F. (1982). Stress, personality and serum cholesterol level. J. Hum. Stress 8: 24-29.

Waldstein, S. R., Manuck, S. B., Bachen, E. A., Muldoon, M. F., and Bricker, P. L. (1990). Anger expression, lipids, and lipoproteins. Poster presentation at the Eleventh Annual Meeting of the Society of Behavioral Medicine, Chicago, April 18-20, p. 102 (abstr.).

Warnick, G. R., and Ables, J. J. (1978). A comprehensive evaluation of the heparin-manganese precipitation procedure for estimating high density lipoprotein cholesterol. J. Lipid Res. 19: $65-76$.

Weidner, G., Sexton, G., McLellarn, R., Connor, S. L., and Matarazzo, J. D. (1987). The role of Type $A$ behavior and hostility in an elevation of plasma lipids in adult women and men. Psychosom. Med. 49: 136-45.

Wilson, W. F. (1989). The epidemiology of hypercholesterolemia. A global perspective. Am. J. Med. 87 (Suppl. 4A): 5S-13S.

Zanchetti, A., Baccelli, G., Mancia, G., and Ellison, D. (1972). Emotion and Cardiovascular System in the Cat. Physiology, Emotion and Psychosomatic Illness, Ciba Foundation Symposium, North Holland, Amsterdam, pp. 201-223. 